\title{
Bedside endoscopy of patients with gastrointestinal bleeding: early does not mean hurried
}

Junseok Park

Department of Internal Medicine, Soonchunhyang University College of Medicine, Seoul, Korea

Received: February 1, 2018 Accepted: February 9, 2018

\section{Correspondence to Junseok Park, M.D. \\ Department of Internal Medi- cine, Soonchunhyang University Hospital Seoul, 59 Daesagwan-ro, Yongsan-gu, Seoul 04401, Korea Tel: +82-2-710-3079 \\ Fax: +82-2-709-9868 \\ E-mail:junspark@schmc.ac.kr}

\section{See Article on Page 304-312}

Critically ill patients can develop gastrointestinal (GI) bleeding during treatment in the intensive care unit (ICU). The treatment approach to GI bleeding is as complex as the reasons for ICU admission.

Stress ulceration has been reported as an important complication in critically ill patients. Inflammation and altered circulation in the splanchnic region can easily reduce the GI mucosal defense mechanisms, triggering ulcerations, and related bleeding [1].

Because of underlying diseases, many critically ill patients use medications that can promote bleeding, such as antiplatelet and anticoagulant agents, as well as corticosteroids. Such patients are at high risk of venous thromboembolism, and prophylactic anticoagulation is recommended [2].

Mechanical ventilation and coagulopathy were identified as independent risk factors for GI bleeding in critically ill patients. Stress ulcer prophylaxis and medical correction are recommended in patients with these risk factors [3].

The optimal timing of endoscopic intervention for such patients is difficult to accurately define, as it depends on clinical features. Generally, endoscopic evaluation within 24 hours of bleeding is recommended, provided that the hemodynamic status is stable. However, endoscopy to control bleeding may be urgently required if GI bleeding is life-threatening [4-7].

GI bleeding is categorized into upper and lower GI tract bleeding to direct patient evaluation and management. Overt symptoms (hematemesis, melena, or hematochezia) may help identify the location of bleeding. However, 10\% to $15 \%$ of patients with severe hematochezia have a bleeding source in the upper GI tract; patients exhibiting hemodynamic instability require upper tract endoscopy to exclude acute upper GI bleeding [8]. Predictors of the outcomes of lower GI bleeding are not as well-established as those of upper GI bleeding, which stops spontaneously in $80 \%$ to $85 \%$ of patients and is associated with a relatively low mortality rate, of $2 \%$ to $4 \%$ [9]. Additionally, bowel preparation should be considered prior to endoscopic intervention for lower GI bleeding [4]. This may delay endoscopy in such patients.

In the current issue, Kim et al. [10] investigated the effectiveness of bedside endoscopy in terms of the diagnosis and treatment of GI bleeding in ICU patients. It is important to ensure optimal timing of bedside endoscopy. The authors mention limitations of the study that were considered to arise from the retrospective design. Howev- 
er, it is also important to review whether other important premises were handled appropriately.

The authors divided patients undergoing endoscopy into two groups, in terms of the timing. This should be analyzed in more detail. Although the authors did not analyze off-duty variables, they mention that endoscopy tended to be delayed during such periods. Unfortunately, no study has yet considered whether bleeding symptoms developing outside of the working hours of endoscopists delay treatment.

Additionally, bowel preparation before colonoscopy requires time. The authors mention that they generally managed patients with $4 \mathrm{~L}$ of oral polyethylene glycol solution (PEG). The reported ideal latency to colonoscopy after completion of bowel preparation using the splitdose method is 3 to 4 hours, and should be less than 8 hours $[11,12]$. With the full-dose PEG method, the recommended latency to surgery is 5 to 6 hours after the last PEG intake [13]. These points should be used to inform the timing of colonoscopy.

The patients enrolled in the present study exhibited inconsistent clinical characteristics. Compared to patients with lower GI bleeding, more patients with upper GI bleeding were managed via early endoscopy (73.9 vs. $52.2 \%, p=0.01$ ). Moreover, patients with upper GI bleeding who were managed via early endoscopy exhibited higher Acute Physiology and Chronic Health Evaluation (APACHE) II scores than did those managed via late endoscopy $(28.6 \pm 9.0$ vs. $24.1 \pm 9.5, p=0.00)$. The APACHE II score reflects mean arterial pressure, heart rate, and the hematocrit level, all of which are closely related to bleeding. Thus, it appears that endoscopy tended to be early in patients with more severe GI bleeding.

In both the upper and lower GI bleeding groups, many patients were on mechanical ventilation. This is closely associated with stress ulceration and GI bleeding of critically ill patients. The authors should analyze the effects of mechanical ventilation separately, comparing the bleeding characteristics and outcomes of ICU patients on and not on ventilation.

As the authors state, many confounding factors may have influenced the results, in addition to clinician subjectivity. However, the results are valuable in terms of the management of critically ill patients with GI bleeding. Consistently, early endoscopic intervention was helpful in patients with upper GI bleeding and the importance of bowel preparation before colonoscopy was reaffirmed by this study. However, the present findings do not devalue the role of early colonoscopy in patients with lower GI bleeding: early endoscopy does not mean hurried endoscopy. Improving the outcomes of patients with GI bleeding requires accurate diagnosis via thoughtful endoscopy; preparation takes time. Also, endoscopy should not be delayed by clinician factors.

\section{Conflict of interest}

No potential conflict of interest relevant to this article was reported.

\section{REFERENCES}

1. van der Voort PH. How to prevent and treat gastrointestinal bleeding in the critically ill patient: a pathophysiological approach. J Emerg Crit Care Med 2017;1:35.

2. Holbrook A, Schulman S, Witt DM, et al. Evidence-based management of anticoagulant therapy: Antithrombotic Therapy and Prevention of Thrombosis, 9th ed: American College of Chest Physicians Evidence-Based Clinical Practice Guidelines. Chest 2012;141(2 Suppl):e152S-e184S.

3. Cook DJ, Fuller HD, Guyatt GH, et al. (1994) Risk factors for gastrointestinal bleeding in critically ill patients. Canadian Critical Care Trials Group. N Engl J Med 1994;330:377-381.

4. Strate LL, Gralnek IM. ACG Clinical Guideline: management of patients with acute lower gastrointestinal bleeding. Am J Gastroenterol 2016;111:459-474.

5. Barkun A, Bardou M, Marshall JK; Nonvariceal Upper GI Bleeding Consensus Conference Group. Consensus recommendations for managing patients with nonvariceal upper gastrointestinal bleeding. Ann Intern Med 2003;139:843-857.

6. Hwang JH, Fisher DA, Ben-Menachem T, et al. The role of endoscopy in the management of acute non-variceal upper GI bleeding. Gastrointest Endosc 2012;75:1132-1138.

7. Gralnek IM, Dumonceau JM, Kuipers EJ, et al. Diagnosis and management of nonvariceal upper gastrointestinal hemorrhage: European Society of Gastrointestinal Endoscopy (ESGE) Guideline. Endoscopy 2015;47:a1-a46.

8. Laine L, Shah A. Randomized trial of urgent vs. elective colonoscopy in patients hospitalized with lower GI bleeding. Am J Gastroenterol 2010;105:2636-2641. 
9. Farrell JJ, Friedman LS. Review article: the management of lower gastrointestinal bleeding. Aliment Pharmacol Ther 2005;21:1281-1298.

10. Kim JH, Kim JH, Chun J, Lee C, Im JP, Kim JS. Early versus late bedside endoscopy for gastrointestinal bleeding in critically ill patients. Korean J Intern Med 2018;33:304312.

11. Seo EH, Kim TO, Park MJ, et al. Optimal preparation-to-colonoscopy interval in split-dose PEG bowel preparation determines satisfactory bowel preparation quality: an observational prospective study. Gastrointest Endosc 2012;75:583-590.

12. Bryant RV, Schoeman SN, Schoeman MN. Shorter preparation to procedure interval for colonoscopy improves quality of bowel cleansing. Intern Med J 2013;43:162-168.

13. Kim TK, Kim HW, Kim SJ, et al. Importance of the time interval between bowel preparation and colonoscopy in determining the quality of bowel preparation for full-dose polyethylene glycol preparation. Gut Liver 2014;8:625-631. 